\title{
Comparative study of polymers and total polar compounds as indicators of refined oil degradation during frying
}

Ibtissem Ben Hammouda ${ }^{1} \cdot$ Gloria Márquez-Ruiz $^{2} \cdot$ Francisca Holgado $^{2} \cdot$ Flavia Freitas $^{3} \cdot$ M. D. R. Gomes Da Silva ${ }^{3}$ Mohamed Bouaziz ${ }^{1,4}$

\begin{abstract}
The aim of this work was to compare the frying stability of refined olive pomace oil alone and blended with refined coconut oil during 60 successive sessions. Frying experiments were carried out at $180{ }^{\circ} \mathrm{C}$ and samples were evaluated by high-performance size-exclusion chromatography (HPSEC), measuring the polymers and polar compounds formed. The tocopherol content was also analyzed. At the end of the frying process, the lowest content of polymeric compounds (PC) and total polar compounds (TPC) were detected for the blend of refined olive pomace oil-refined coconut oil (ROPO/RCO) with $13.20 \%$ and $25 \%$, respectively, compared to refined olive pomace oil (ROPO) pure with $16.9 \%$ and $34.5 \%$, respectively. Hence, the present study based on PC and TPC as best quality indicators of frying oil degradation indicated that the frying behavior of ROPO pure significantly improved by the blending application with RCO and showed a higher chemical stability.
\end{abstract}

\section{Graphical abstract}

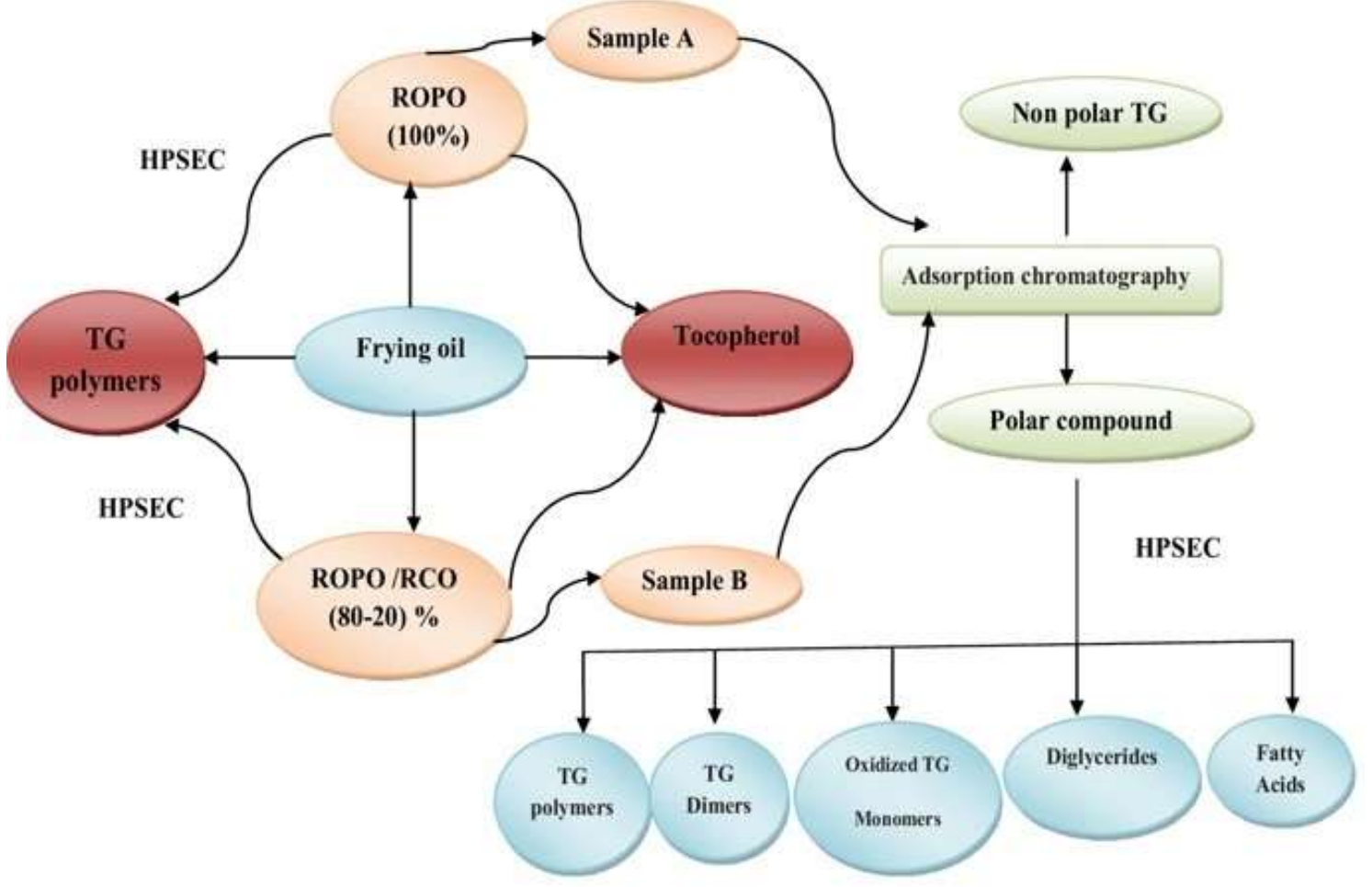




\title{
Keywords
}

Deep-fat frying $\cdot$ Total polar compounds $\cdot$ Polymeric compounds $\cdot$ Solid-phase extraction.

\author{
Abbreviations \\ ROPO: Refined olive-pomace oil \\ RCO: Refined coconut oil \\ HPSEC: High-performance size-exclusion chromatography \\ SFA: Saturated fatty acids \\ PUFA: Polyunsaturated fatty acids \\ TPC: Total polar compounds \\ MAG: Monoacylglycerols \\ DAG: Diacylglycerols \\ ox-TGM: Oxidized triglyceride monomers \\ TGO: Triglyceride oligomers \\ TGD: Triglyceride dimers
}

\section{Introduction}

Frying process is a fast, convenient, and energy-efficient cooking procedure that increases palatability and provides crust formation together with pleasing flavors and odors [1]. This process is a multifunctional operation of food transformation of high complexity that involves challenges to oil quality $[2,3]$. The presence of oxygen, the moisture content of the food, the higher oil temperature and the leaching of components from the food result in the formation of a high variety of products with different polarity, stability and molecular weight [3, 4]. These components include TAG dimers, polymers, oxidized TAGs, and volatile compounds, all of which contribute to degradation of the frying oil [3].

The stability of frying oil is a crucial factor in the quality and health effect of both fried foods and oils [5]. Therefore, it is imperative to assess oil quality to avoid the use of deteriorated oil [5] by the best methods recommended for evaluating frying degradation, which are the determination of polar compounds [6] and the determination of polymers [7]. Besides, essays on deep-fat frying in a standard fryer remain the best method to test frying performance of oils [8]. To obtain broader information on fat alteration, polar compound (PC) content and the level of different thermal oxidation and hydrolytic compounds are good quality indicators of frying fats' alteration [9]. Maximum levels of $25 \%$ polar compounds or $16 \%$ polymers have been established as the upper limits of oil degradation for human consumption by most countries adopting regulations on frying fats and oils $[4,10]$. 
Several studies on comparative performance of different oils and fats in the frying process have been reported $[4,11,12]$. Oils with high levels of polyunsaturated fatty acids (PUFA) are not proper for frying due to their fatty acid composition, with a higher tendency to oxidation $[4,11$, $13]$. Blending is one of the strategies used to enhance the frying stability [12, 14], which improves oil functionalities and adjusts the fatty acid profile to optimal levels for frying applications. Refined olive pomace oil, like the majority of vegetable oils, contains a variety of minor components such as tocopherols which are beneficial to oil stability [15] and has already been used in preparation of an ideal frying oil mixture [16]. Ben Hammouda et al. demonstrated that blends of monounsaturated oil like refined olive pomace oil (ROPO) with refined oil rich in saturated fatty acids like refined palm oil (RPO) (25:75) revealed better frying performance compared to other blends [17]. Likewise, according to previous studies, coconut oil is very stable to oxidative deterioration when exposed to atmospheric oxygen due to the higher degree of saturated fatty acids (about 92\%) [18].

The aim of the present research work is to evaluate the quality of refined olive pomace oil (ROPO) during deep-frying at $180{ }^{\circ} \mathrm{C}$, and to enhance the potential of this frying oil by adding $20 \%$ of refined coconut oil (RCO), which is rich in saturated fatty acid and hence should improve the frying and the oxidative stability during heating procedure. To our knowledge, no study has been reported so far on blending refined olive pomace oil with refined coconut oil to formulate new frying oils.

\section{Materials and methods}

\section{Oil samples}

The studied oils during the 60 deep-frying sessions, which were obtained from the National Oil Office of Sfax, Tunisia, were refined olive-pomace oil (ROPO) and refined coconut oil (RCO). The studied oil blend (ROPO/RCO) was prepared in the volume ratio of 80:20, after comparison of oxidative stability with other proportions using the 743 Rancimat methods (data not shown). Blending the polyunsaturated oils with more saturated or monounsaturated oils is a potential solution to improve oil stability [14].

\section{Frying process}

Spunta variety potatoes from Tunisia were peeled, cut approximately in uniform pieces $(5 \mathrm{~cm}$ long and $0.5 \mathrm{~cm}$ thick), washed, and wiped before the frying experiments. The latter was conducted in the same manner as the actual household cooking process. For deep-frying, an electric common domestic fryer type (MOULINEX mega fryer-White-Power: 2100 wattsAnti-odor filter—Anti-skid feet—Oil capacity: 3L_-Adjustable thermostat—Removable bowl— Size: $35 \times 29 \times 32 \mathrm{~cm}$-Weight: $4.21 \mathrm{~kg}$ ) was used. It is equipped with a thermostat and supplied 
with an inert cross-linked steel wire mesh that allows the food to be dipped into the oil without coming in contact with the fryer's inner surface. In every frying session, $200 \mathrm{~g}$ of potatoes was deep-fried for $9 \mathrm{~min}$ at $180{ }^{\circ} \mathrm{C}$ in $2.7 \mathrm{~L}$ of refined oil, without replenishment.

The fryer lid was closed when the cooling started. After cooling $(\approx 30 \mathrm{~min})$, the frying process was repeated 60 times using new potatoes in the same oil. The samples of the fresh, refined oils together with those sampled after each ten successive deep-frying sessions were stored in sealed dark glass bottles at $-20^{\circ} \mathrm{C}$ until analyses.

\section{Fatty acid composition}

Fatty acid composition was determined by the IUPAC Standard methods [19]. Following derivatization to FAMEs with $2 \mathrm{M} \mathrm{KOH}$ in methanol, FAMEs were analyzed by GC- 6850 (Agilent Technologies, Palo Alto, CA, USA) equipped with a FID detector. FAMEs $(c=50$ $\mathrm{mg} / \mathrm{mL}$ in hexane, volume injected $=2 \mu \mathrm{L}$ ) were separated using HP Innowax capillary column ( $30 \mathrm{~m} \times 0.25 \mathrm{~mm}$ id, $0.25 \mu \mathrm{m}$ film thickness). The temperature program used was $180{ }^{\circ} \mathrm{C}$ for 2 min, followed by a $3{ }^{\circ} \mathrm{C} / \mathrm{min}$ increase to $230{ }^{\circ} \mathrm{C}$ and held there for $20 \mathrm{~min}$. The temperatures of the injector and detector were held at $250^{\circ} \mathrm{C}$. Hydrogen was the carrier gas at a flow rate of 1 $\mathrm{mL} / \mathrm{min}$ with a split ratio of 1:40. Proportions of fatty acids were determined as weight percentage $(\%)$.

\section{Determination of total polar compounds}

Total polar compounds (TPC) were determined by means of rapid test instrument (Testo 270 Deep-frying Oil Tester, Testo Inc., Germany) in the oil blend samples during the frying process. Tests were performed according to the manufacturer's guidelines. A sensor based on parallel plate capacitor was immersed in hot oil at frying temperature. The sensor took about $10 \mathrm{~s}$ to get a stable reading. The TPC percentages along with the temperature were displayed on the screen of the instrument [4].

\section{Quantitation of triacylglycerol polymers}

Aliquots of $50 \mathrm{mg}$ oil were dissolved in $1 \mathrm{~mL}$ tetrahydrofuran for direct analysis by HPSEC. A chromatograph equipped with a Rheodyne $7725 \mathrm{i}$ injector with a $10-\mu \mathrm{L}$ sample loop, a Knauer 120 HPLC pump (Knauer, Berlin, Germany) and a Merck L-7490 refractive index detector (Merck, Darmstadt, Germany) was used. The separation was performed on two 100 and 500 Ultrastyragel columns $(25 \mathrm{~cm} \times 0.77 \mathrm{~cm}$ i.d. $)$ packed with porous, highly cross-linked styrenedivinylbenzene copolymers (particle size $5 \mu \mathrm{m}$ ) (Hewlett-Packard, Avondale, PA, USA) connected in series, with tetrahydrofuran $(1 \mathrm{~mL} / \mathrm{min})$ as the mobile phase, according to IUPAC Standard Method 2.508 [20]. The groups of compounds quantified were dimers and higher oligomers of triacylglycerols [21]. The sum of dimers and higher oligomers will be referred to as polymers.

Eur Food Res Technol 245, 967-976 (2019). https://doi.org/10.1007/s00217-018-3202-5 


\section{Quantitation and distribution of total polar compounds}

The content of total polar compounds was determined gravimetrically according to IUPAC Standard Method 2.507 [20] with slight modifications. Thus, the non-polar and polar fractions were separated from $1 \mathrm{~g}$ of oil by silica column chromatography. The non-polar fraction, which contains the non-polar TAG, was eluted with $150 \mathrm{~mL}$ of $\mathrm{n}$-hexane/diethyl ether (90:10, v/v). A second fraction, which comprises the total polar compounds, was eluted with $150 \mathrm{~mL}$ of diethyl ether. The solvents were evaporated and the contents of the non-polar and polar fractions were determined gravimetrically. The efficiency of the separation was checked out by thin-layer chromatography using hexane/diethyl ether/ acetic acid (80:20:1, v/v/v) for development of plates and exposing to iodine vapor to reveal the spots. The polar fraction was analyzed by HPSEC to determine the content of oligomeric, dimeric and oxidized monomeric triglycerides (TG), as well as diglycerides (DG) and fatty acids. A chromatograph equipped with a Rheodyne $7725 \mathrm{i}$ injector with a $10 \mu \mathrm{L}$ sample loop, a Knauer 1200 HPLC pump (Knauer, Germany) and a Merck refractive index detector was used. The separation was performed on two 100 and $500 \AA$ PL gel columns $(30 \mathrm{~cm} \times 0.75 \mathrm{~cm}$ I.D. $)$ packed with porous, highly cross-linked polystyrene-divinylbenzene copolymers (film thickness $5 \mu \mathrm{m}$ ) (Agilent Technologies) connected in series. Tetrahydrofuran $(1 \mathrm{~mL} / \mathrm{min})$ was used as the mobile phase and samples were analyzed at concentrations between 15 and $20 \mathrm{mg} / \mathrm{mL}$ in tetrahydrofuran.

\section{Tocopherol content}

Approximately $0.10 \mathrm{~g}$ of the oil sample was accurately weighed in a $50-\mathrm{mL}$ polypropylene centrifugation tube and then $10.0 \mathrm{~mL}$ of methanol was added. The mixture was thoroughly vortexed for $1 \mathrm{~min}$, sonicated at water bath for $30 \mathrm{~min}$ and centrifuged at $8000 \mathrm{rpm}$ for $3 \mathrm{~min}$. An aliquot of the supernatant was transferred into a vial and then analyzed by the GC-FID system, according to Zhang et al. [22].

\section{Results and discussion}

\section{Fatty acid composition}

The fatty acid compositions of initial refined olive pomace oil (ROPO) and blended with refined coconut oil (ROPO/ RCO) are given in Table 1.

The fatty acids identified were palmitic acid (C16:0), stearic (C18:0), oleic (C18:1), linoleic (C18:2), linolenic (C18:3) and arachidic acids (C20:0). The most predominant fatty acids in both fresh oils (ROPO) and (ROPO/RCO) are palmitic acid (15.70-20.08\%), oleic acid (57.51$56.38 \%$ ) and linoleic acid (20.80-15.63\%). The addition of $20 \%$ of RCO (rich in saturated fatty acid) to ROPO decreased the content of linoleic and linolenic acids (the fatty acids most susceptible to oxidation) from 20.80 to $15.63 \%$ and 1.00 to $0.56 \%$, as well increased the palmitic 
acid content (the fatty acids most stable) from 15.70 to $20.08 \%$ of the pure and blended oils, respectively. Thus, the composition of fatty acids clearly improved in the mixture with the refined coconut oil (RCO).

According to our previous results [14], the data showed that the amount of PUFAs such as the linoleic and linolenic acids decreased gradually after 60 sessions of frying in both oils (ROPO pure and $\mathrm{ROPO} / \mathrm{RCO}$ ) ranging between $16.95 \%$ and $0.64 \%$, respectively, whereas, varying between $12.94 \%$ and $0.40 \%$, respectively. Consequently, a relative increase in the percentages of SFAs such as the palmitic acid was observed. It is well known that oils rich in SFAs are characterized by high thermal stability. In fact, the lowest value of C16:0 was observed for the ROPO pure with $17.24 \%$, while the highest one for the blended oil with $20.87 \%$ after 60 sessions of frying.

\section{Determination of total polar compounds and polymers}

Table 2 shows the results obtained for total polar compounds and polymers in duplicate samples of oils heated at $180^{\circ} \mathrm{C}$ for 60 successive sessions of frying, under the conditions described in the experimental part. Total polar compounds are considered as an excellent oil degradation indicator because it refers to all degraded products [23]. These degradation compounds with a high variety of different polarity, stability and molecular weight, including polymeric triacylglycerols (PTAG), oxidized-triacylglycerols (ox-TAG), oxidized-monoacylglycerols (ox-MAG), diacylglycerols (DAG), monoacylglycerols (MAG) and FFAs are formed as a result of thermal oxidation reactions or hydrolysis of triglycerides during frying procedures [17].

Many countries have established regulatory limits for TPC in frying oils and considered $25 \%$ or $16 \%$ polymers as limits $[14,24]$. Based on the rapid measurements of TPC using the Testo 270, results revealed that both the duration of heating and the nature of oils affect the total polar compounds (Table 2). Initially, the TPC level in the pure ROPO and blend ROPO/RCO was 9\% and $9.5 \%$, while the content of polymeric compounds was $1.9 \%$ and $0.9 \%$, respectively. Faster increment of TPC was found for refined olive pomace oil, from 9 to $34.5 \%$, than in the blend ROPO/RCO (from 9.5 to $25 \%$ ) after 60 successive deep-frying sessions of potato fries at $180{ }^{\circ} \mathrm{C}$. Indeed, the limit of TPC for human consumption is estimated for ROPO after 40 sessions, whereas it was after 60 sessions for the blend. Therefore, the blend ROPO/RCO showed excellent performance. Polymeric compounds are a group of components with different structures consisting of polar and non-polar lipid compounds in frying oil [25] which were determined by high-performance size exclusion chromatography (HPSEC) [26]. Results showed a higher increase of a polymeric compound for ROPO, from 3.20 to $16.90 \%$, after 60 sessions of frying than for the ROPO/RCO blend, from 3.20 to $13.20 \%$, in this latter case below the limit of $12-$ $16 \%$ for use of frying fats and oils [24]. 
As expected, according to our results (Table 2), TPC and PC are in compliance. Their content increased gradually with frying sessions as previously $[4,11,27,28]$. This increase is due to oxidation and thermal reactions during frying [29] which lead to the formation of high molecular weight compounds $[4,30]$. Under the same conditions, the blend ROPO/RCO showed lower content of total polar compounds and polymeric compounds during frying than that observed for ROPO, which can be explained by the high content of PUFAs in the latter, in agreement with previous studies $[4,31]$.

Also, two oil samples were chosen (the blend ROPO/RCO after 60 sessions of frying and the ROPO after 40 sessions of frying), when their TPC levels were close to the maximum value for human consumption (25\%) [17]. Similar distributions of groups of compounds were found in both samples (Table 3).

The distribution of polar compounds showed the quantitative importance of TGP (TGO + TGD) and oxTGM in the thermoxidized samples [26]. This can be easily observed by comparing chromatograms in Figs. 1 and 2.

In brief, results showed that the blend ROPO/RCO was more stable than ROPO during frying.

\section{Limits of detection and quantification}

The parameters of the regression equations, LOD, LOQ, and the calibration range are summarized in Table 4. The instrumental detection limits (LODs) and quantification limits (LOQs) were estimated using the signal-to-noise ratio $(\mathrm{S} / \mathrm{N})$, determined as the lowest concentration producing a signal-to-noise ratio of at least 3:1 and 10:1, respectively.

The instrumental LODs and LOQs are in the low $\mathrm{mg} / \mathrm{kg}$ range, ranging from 0.15 to $0.59 \mathrm{mg} / \mathrm{kg}$ and 0.50 to $1.97 \mathrm{mg} / \mathrm{kg}$, respectively.

\section{Determination of tocopherols}

Tocopherols are antioxidants that act as radical scavengers to decelerate the propagation phases of oxidative degradation [32]. The three isoforms of tocopherols ( $\alpha$-tocopherol, $\beta$-tocopherol and $\gamma$-tocopherol) were identified in the oil samples (Fig. 3). These isoforms are commonly present in plant-derived edible oils [33].

Initially, $\alpha$ tocopherol was present as a major compound in both oils, ROPO and ROPO/RCO, with $240.11 \mathrm{mg} / \mathrm{kg}$ and $208.50 \mathrm{mg} / \mathrm{kg}$, respectively (Table 5), while the other isoforms showed a lower content, with (3.30-4.61) $\mathrm{mg} / \mathrm{kg}$ and (3.81-12.6) $\mathrm{mg} / \mathrm{kg}$ for $\boldsymbol{\gamma}$ and $\boldsymbol{\beta}$ tocopherols, respectively.

According to the results obtained, the heating procedure and the frying time affected the tocopherol content in the oil samples by a significant degradation during frying. Indeed, the pure oil (ROPO) which contained the highest level of total tocopherol with $248.02 \mathrm{mg} / \mathrm{kg}$ at fresh state underwent an important reduction $(p<0.05)$ during the frying process. Total tocopherol content 
was reduced by $94.40 \%$, from 248.02 to $14.02 \mathrm{mg} / \mathrm{kg}$, after 40 successive sessions of frying in ROPO and by $90.22 \%$, from 224.91 to $21.94 \mathrm{mg} / \mathrm{kg}$, in ROPO/RCO. Total tocopherols degraded sharply in both oils and were practically exhausted after 60 successive deep-frying sessions.

\section{Conclusion}

The present study highlights the effectiveness of blending in retarding oil degradation during frying. Progression of oxidation was basically followed by detecting polar and polymeric compounds which is considered as representative of oil degree degradation. The results clearly indicated that the frying performance of ROPO significantly improved by the blending with RCO and revealed a great resistance to oxidative deterioration after 60 successive sessions of frying as compared to ROPO pure. Thus, this research may aid in the evaluation and the selection of frying oils as well increases their shelf life in the fast-food industries and in domestic food preparation applications.

\section{Acknowledgements}

The authors would like to thank the "Ministère de l'Enseignement Supérieur et de la Recherche Scientifique" (Laboratory LR14ES08), Tunisia, for its financial support for this research work. The authors acknowledge also National Funds through Ministry of Higher Education-Tunisia for financing MedOOmics Project_-"Mediterranean Extra Virgin Olive Oil Omics: profiling and fingerprinting"- “Arimnet2/0001/2015".

\section{Compliance with ethical standards}

Conflict of interest: The authors declare no competing financial interest.

Compliance with ethics requirements: This article does not contain any studies with human or animal subjects. 


\section{References}

1. Gertz C (2014) Fundamentals of the frying process. Eur J Lipid SciTechnol 116:669-674

2. Kalogianni EP, Papastergiadis E (2014) Crust pore characteristics and their development during frying of French fries. J Food Eng 120:175-182

3. Magda A, Hamdy Z, Agnieszka K (2016) The effect of pan frying on thermo-oxidative stability of refined rapeseed oil and professional blend. J FoodSciTechnol 53:712-720

4. Zribi A, Jabeur H, Matthäus B, Bouaziz M (2016) Quality control of refined oils mixed with palm oil during repeated deep-frying using FTNIRS, GC, HPLC, and multivariate analysis. Eur J Lipid Sci Technol 118:512-523

5. Das AK, Babylatha R, Pavithra AS, Khatoon S (2013) Thermal degradation of groundnut oil during continuous and intermittent frying. J Food Sci Technol 50:1186-1192

6. IUPAC (1987) International union of pure and applied chemistry, standard methods for the analysis of oils, fats and derivatives, 7 th edn. Blackwell, Oxford

7. IUPAC (1992) International union of pure and applied chemistry, standard methods for the analysis of oils, fats and derivatives, $1^{\text {st }}$ Supplement to the 7 th Edn. Blackwell, Oxford

8. Felix A, Roman P (2011) Rapid assessment of frying performance using small size samples of oils/fats. J Am Oil Chem Soc 88:1867-1873

9. Barrera-Arellano D, Ruiz-Méndez MV, Velasco J, Márquez-Ruiz G, Dobarganes MC (2002) Loss of tocopherols and formation of degradation compounds at frying temperatures in oils differing in unsaturation degree and natural antioxidant content. J Sci Food Agric 82:1696-1702

10. Firestone D (2007) In: Erickson MD (Ed) Deep frying: chemistry nutrition and practical applications. 2nd Edn., AOCS, Champaign. pp. 373-385

11. Zribi A, Jabeur H, Aladedunye F, Rebai A, Matthäus B, Bouaziz M (2014) Monitoring of quality and stability characteristics and fatty acid compositions of refined olive and seed oils during repeated pan and deep-frying using GC, FT-NIRS, and chemometrics. J Agric Food Chem 62:10357-10367

12. Abdulkarim SM, Myat MW, Ghazali HM, Roselina K, Abbas KA (2010) Sensory and physicochemical qualities of palm olein and sesame seed oil blends during frying of banana chips. J Agric Sci 2:18-29

13. Ramadan MF, Wahdan KMM (2012) Blending of corn oil with black cumin (Nigella sativa) and coriander (Coriandrum sativum) seed oils: impact on functionality, stability and radical scavenging activity. Food Chem 132:873-879

14. Ben Hammouda I, Triki M, Matthäus B, Bouaziz M(2018) A comparative study on formation of polar components, fatty acids and sterols during frying of refined olive pomace oil pure and its blend coconut oil. J Agric Food Chem. https ://doi.org/10.1021/acs.jafc.7b051 63

15. Rossi M, Alamprese C, Ratti S (2007) Tocopherols and tocotrienols as free radical-scavengers in refined vegetable oils and their stability during deep-fat frying. Food Chem 102:812-817

16. Erdim B, Emin Y (2010) Comparison of the frying stability of sunflower and refined olive pomace oils with/without adsorbent treatment. J Am Oil Chem Soc 87:1145-1153

17. Ben Hammouda I, Zribi A, Ben Mansour A, Matthäus B, Bouaziz M (2017) Effect of deep-frying on 3-MCPD esters and glycidyl esters contents and quality control of refined olive pomace oil blended with refined palm oil. Eur Food Res Technol 243:1219-1227

18. Faiza S, Debnath S (2016) Molecular finger printing of nutra-coconut oil with improved health protective phytoceuticals and its efficacy as frying medium. J Am Oil Chem Soc 93:1301-1310

19. IUPAC (1992) Standard methods for the analysis of oils, fats and derivatives, 7th edn. International Union of Pure and Applied Chemistry, Blackwell Scientific, Oxford

20. Márquez-Ruiz G, Dobarganes MC (2007) High-performance size exclusion chromatography for lipid analysis in organic media. In: Mossoba MM, Kramer JKG, Brenna JT, McDonald RE (eds) Lipid analysis and lipidomics. New techniques and applications. AOCS Press, Champaign, pp 205-238

21. Dobarganes MC, Velasco J, Dieffenbacher A (2000) The determination of polar compounds, polymerised triacylglycerols, oxidized triacylglycerols and diacylglycerols in fats and oils. Pure Appl Chem 72:1563-1575 22. Zhang R, Shen W, Wei X, Zhang F, Shen C, Wu B, ...Deng X (2016) Simultaneous determination of tocopherols and tocotrienolsin vegetable oils by GC-MS. Anal Methods 8:7341-7346

23. Urbancic S, Kolar MH, Dimitrijevic D, Vidrih R (2013) Stabilisation of sunflower oil and reduction of acrylamide formation of potato rosemary extract during deep-fat frying. LWT Food Sci Technol 57:671-678 24. Gloria MR, Victoria RM, Joaquín V (2014) Antioxidants in frying: analysis and evaluation of efficacy. Eur J Lipid Sci Technol 116:1441-1450

Eur Food Res Technol 245, 967-976 (2019). https://doi.org/10.1007/s00217-018-3202-5 
25. Abidi SL, Rennick KA (2003) Determination of nonvolatile components in polar fractions of rice bran oils. J Am Oil Chem Soc 80:1057-1062

26. Fatma NA, Ayça N, Fatma D, Huseyin KA (2016) Study on monitoring of frying performance and oxidative stability of cottonseed and palm oil blends in comparison with original oils. Int J Food Prop.

https://doi.org/10.1080/10942 912.2016.11775 44

27. Tabee E, Jägerstad M, Dutta PC (2009) Frying quality characteristics of French fries prepared in refined olive oil and palm olein. J Am Oil Chem Soc 2009,86: 885-893

28. Farhoosh R, Tavassoli-Kafrani MH (2010) Polar compounds distribution of sunflower oil as affected by unsaponifiable matters of bene hull oil (BHO) and tertiary-butylhydroquinone (TBHQ) during deep-frying. Food Chem 122:381-385

29. Zhang Q, Saleh ASM, Chen J, Shen Q (2012) Chemical alterations taken place during deep-fat frying based on certain reaction products: a review. Chem Phys Lipids 165:662-681

30. Gloria MR, Francisca H, Victoria RM, Joaquín V, Carmen GM (2016) Oxidation of a functional, CLA rich oil: determination of volatile and nonvolatile compounds. Eur Food Res Technol 242:1993-2000

31. Ali Rehab FM, ElAnany AM (2012) Physicochemical studies on sunflower oil blended with cold pressed tiger nut oil during the deep frying process. Grasas Aceites 63:455-465

32. Sanchez-Muniz FJ, Botega DZ, di Lorenzo L, Marmesat S et al (2007) A non-extractable condensed-tannins fiber reduces thermal oxidation in oils at frying temperature. Eur J Lipid Sci Technol 109:1218-1225

33. Elisia I, Young JW, Yuan YV, Kitts DD (2013) Association between tocopherol isoform composition and lipid oxidation in selected multiple edible oils. Food Res Int 52:508-514

\section{Affiliations}

Ibtissem Ben Hammouda ${ }^{1}$. Gloria Marquez-Ruiz ${ }^{2}$ Francisca Holgado ${ }^{2}$ Flavia Freitas ${ }^{3} \cdot$ M. D. R. Gomes Da Silva ${ }^{3} \cdot$ Mohamed Bouaziz $^{1,4}$

* Mohamed Bouaziz mohamed.bouaziz@fsg.rnu.tn

${ }^{1}$ Laboratoire d'Électrochimie et Environnement, École Nationale d'Ingénieurs de Sfax,

Université de Sfax, B.P. 1173, 3038 Sfax, Tunisia

${ }^{2}$ Instituto de Ciencia y Tecnología de Alimentos y Nutrición, Consejo Superior de

Investigaciones Científicas (ICTAN-CSIC), Madrid, Spain

${ }^{3}$ Departamento de Química, Faculdade de Ciências e Tecnologia, LAQV, REQUIMTE, Universidade Nova de Lisboa, 2829-516 Caparica, Portugal

${ }^{4}$ Institut Supérieur de Biotechnologie de Sfax, Université de Sfax, B.P. 1175, 3038 Sfax, Tunisia. 
Table 1 Fatty acid composition of ROPO and ROPO/RCO (80:20) blend before deep frying.

\begin{tabular}{lcccc}
\hline $\begin{array}{l}\text { Fatty acid } \\
\text { composition, } \mathrm{wt} \%\end{array}$ & ROPO T0 & ROPO after 60 DF & ROPO/RCO T0 & ROPO/RCO after 60 DF \\
\hline C6:0 & & - & $0.06 \pm 0.00$ & $0.03 \pm 0.00$ \\
C8:0 & & - & $0.62 \pm 0.01$ & $0.46 \pm 0.02$ \\
C10:0 & - & $0.36 \pm 0.01$ & $0.31 \pm 0.01$ \\
C12:0 & - & $2.50 \pm 0.04$ & $2.59 \pm 0.01$ \\
C14:0 & $0.02 \pm 0.00$ & $0.03 \pm 0.00$ & $1.09 \pm 0.01$ & $1.16 \pm 0.01$ \\
C16:0 & $15.70 \pm 0.30$ & $17.24 \pm 0.10$ & $20.08 \pm 0.10$ & $20.87 \pm 0.12$ \\
C16:1 & $0.80 \pm 1.10$ & $1.77 \pm 0.02$ & $1.55 \pm 0.00$ & $1.59 \pm 0.01$ \\
C17:0 & $0.10 \pm 0.00$ & $0.07 \pm 0.00$ & $0.06 \pm 0.01$ & $0.07 \pm 0.00$ \\
C17:1 & $0.11 \pm 0.10$ & $0.09 \pm 0.00$ & $0.07 \pm 0.00$ & $0.07 \pm 0.00$ \\
C18:0 & $3.01 \pm 0.00$ & $3.21 \pm 0.02$ & $3.72 \pm 0.02$ & $3.89 \pm 0.01$ \\
C18:1 & $57.51 \pm 0.60$ & $59.13 \pm 0.35$ & $56.38 \pm 0.00$ & $54.84 \pm 0.34$ \\
C18:2 & $20.80 \pm 0.20$ & $16.95 \pm 0.23$ & $15.63 \pm 0.02$ & $12.94 \pm 0.16$ \\
C18:3 & $1.00 \pm 0.00$ & $0.64 \pm 0.00$ & $0.56 \pm 0.00$ & $0.40 \pm 0.01$ \\
C20:0 & $0.51 \pm 0.00$ & $0.56 \pm 0.00$ & $0.42 \pm 0.00$ & $0.49 \pm 0.00$ \\
C20:1 & $0.30 \pm 0.00$ & $0.32 \pm 0.00$ & $0.24 \pm 0.01$ & $0.30 \pm 0.01$ \\
\hline
\end{tabular}

Each value represents the mean of three determinations $(n=3) \pm$ standard deviation $R O P O$ refined olive-pomace oil, $R C O$ refined coconut oil, $D F$ deep-frying 
Table 2 Evolution of total polar compound (\%) and polymer (\%) formation in ROPO and $\mathrm{ROPO} / \mathrm{RCO}(80: 20) \%$ during deep-frying at $180^{\circ} \mathrm{C}$

\begin{tabular}{lcccc}
\hline \multirow{2}{*}{$\begin{array}{l}\text { Session } \\
\text { numbers }\end{array}$} & \multicolumn{2}{c}{ ROPO $100 \%$} & \multicolumn{2}{c}{ ROPO/RCO (80-20\%) } \\
\cline { 2 - 5 } & $\begin{array}{l}\text { Total polar } \\
\text { compound }\end{array}$ & Polymers & $\begin{array}{l}\text { Total polar } \\
\text { compound }\end{array}$ & Polymers \\
\cline { 2 - 5 } & Mean SD & Mean SD & Mean SD & Mean SD \\
\hline 0 & $9 \pm 0.5$ & $1.9 \pm 0.45$ & $9.5 \pm 0.5$ & $0.9 \pm 0.18$ \\
10 & $12 \pm 0.5$ & $3.2 \pm 0.40$ & $11 \pm 0.0$ & $3.2 \pm 0.12$ \\
20 & $15.5 \pm 0.5$ & $6.4 \pm 0.67$ & $12.5 \pm 0.5$ & $3.9 \pm 0.46$ \\
30 & $20.5 \pm 0.5$ & $9.6 \pm 0.76$ & $14.5 \pm 0.5$ & $6.3 \pm 1.38$ \\
40 & $25.5 \pm 0.5$ & $11.4 \pm 0.82$ & $17 \pm 0.5$ & $5.9 \pm 0.55$ \\
50 & $29.5 \pm 0.5$ & $14.6 \pm 0.81$ & $20 \pm 0.0$ & $9.3 \pm 0.97$ \\
60 & $34.5 \pm 0.0$ & $16.9 \pm 1.04$ & $25 \pm 0.0$ & $13.2 \pm 0.21$ \\
\hline
\end{tabular}

Data are expressed as means \pm standard deviations $(n=2)$

$R O P O$ refined olive-pomace oil, $R O P O / R C O$ refined olive-pomace oil/refined coconut oil 
Table 3 Polar compound content and distribution (wt \%) in refined olive pomace oil (ROPO) and $\mathrm{ROPO} / \mathrm{RCO}(80: 20)$ during the frying process at $180^{\circ} \mathrm{C}$

\begin{tabular}{lcccccc}
\hline Samples & Polar & \multicolumn{5}{c}{ Polar compound distribution } \\
\cline { 3 - 7 } & \begin{tabular}{c} 
compounds \\
\cline { 3 - 7 }
\end{tabular} & TGO & TGD & oxTGM & DG & FFA \\
\hline ROPO/RCO 60 & 21.58 & 2.37 & 6.70 & 7.84 & 4.39 & 0.28 \\
ROPO 40 & 22.15 & 2.93 & 7.13 & 7.91 & 3.71 & 0.47 \\
\hline
\end{tabular}

ROPO/RCO 60 refined pomace oil/refined coconut oil (80-20\%) after 60 sessions of frying, $R O P O 40$ refined pomace oil (100) after 40 sessions of frying, $T G O$ triglyceride oligomers, $T G D$ triglyceride dimers, oxTGM oxidized triglyceride monomers, $D G$ diglycerides, $F F A$ fatty acid 
Table 4 Regression equations, correlation coefficients $\left(R^{2}\right)$, limits of detection (LODs) and quantification (LOQs) of the proposed method

\begin{tabular}{ccccc}
\hline Tocopherol & Regression equations & $\mathrm{R}^{2}$ & LODs mg/kg & LOQs mg/kg \\
\hline$\delta$ & $Y=16.086 \mathrm{x}-0.0801$ & 0.95 & 0.59 & 1.00 \\
$\beta$ & $Y=42.13 \mathrm{x}-0.1716$ & 0.94 & 0.39 & 1.30 \\
$\gamma$ & $Y=33.948 \mathrm{x}-0.1784$ & 0.94 & 0.15 & 0.50 \\
$\alpha$ & $Y=28.793 \mathrm{x}-0.1243$ & 0.94 & 0.35 & 1.97 \\
\hline
\end{tabular}

$Y$ peak area of the quantitative ion of the analyte, $X$ mass concentration of the analyte, $\mathrm{mg} / \mathrm{kg}$. 
Table 5 Tocopherol content (mg/kg) of refined oils (ROPO) and (ROPO/RCO) during frying

\begin{tabular}{|c|c|c|c|c|c|c|c|c|}
\hline $\mathrm{T}$ & $\mathrm{ROPO}_{\mathrm{T} 0}$ & $\mathrm{ROPO}_{20}$ & $\mathrm{ROPO}_{40}$ & $\mathrm{ROPO}_{60}$ & $\mathrm{ROPO} / \mathrm{RCO}_{\mathrm{T} 0}$ & $\mathrm{ROPO} / \mathrm{RCO}_{20}$ & $\mathrm{ROPO} / \mathrm{RCO}_{40}$ & $\mathrm{ROPO} / \mathrm{RCO}_{60}$ \\
\hline$\delta$ & nd & nd & nd & nd & nd & nd & nd & nd \\
\hline$\beta$ & $3.30 \pm 0.01 \mathrm{aB}$ & $2.81 \pm 0.02 \mathrm{bB}$ & $1.32 \pm 0.02 \mathrm{cB}$ & nd & $3.81 \pm 0.02 \mathrm{aA}$ & $2.50 \pm 0.01 \mathrm{bA}$ & $1.82 \pm 0.02 \mathrm{cA}$ & nd \\
\hline$\gamma$ & $4.61 \pm 0.02 \mathrm{aB}$ & $3.52 \pm 0.01 \mathrm{bB}$ & $1.20 \pm 0.01 \mathrm{cB}$ & nd & $12.60 \pm 0.05 \mathrm{aA}$ & $5.72 \pm 0.02 \mathrm{bA}$ & $3.01 \pm 0.03 \mathrm{cA}$ & nd \\
\hline$\alpha$ & $240.11 \pm 0.85 \mathrm{aA}$ & $50.20 \pm 0.09 \mathrm{bB}$ & $11.50 \pm 0.05 \mathrm{cB}$ & nd & $208.50 \pm 0.49 \mathrm{aB}$ & $81.22 \pm 0.06 \mathrm{bA}$ & $17.11 \pm 0.04 \mathrm{cA}$ & nd \\
\hline Total & 248.02 & 56.53 & 14.02 & nd & 224.91 & 89.44 & 21.94 & nd \\
\hline
\end{tabular}

$T$ tocopherols, $\alpha$ alpha, $\gamma$ gamma, $\beta$ beta, $\delta$ delta, $R O P O$ refined olive-pomace oil pure, $R O P O / R C O$ refined olive-pomace oil/refined coconut oil (80:20)

Each value represents the average of three determinations $(n=3) \pm$ standard deviation. Different lowercase letters $(\mathrm{a}, \mathrm{b}$ and $\mathrm{c})$ in the same line indicate significant differences $(p<0.05)$ for the same refined oil mixture at different times. Different capital letters (A and B) in the same row indicate significant differences $(p<0.05)$ for the different refined oil mixtures at the same time 


\section{FIGURE CAPTIONS}

Fig. 1 Distribution of polar compounds in ROPO (100:0) after 40 sessions of frying based directly on HPSEC (A) and on combination of solid-phase extraction (SPE) and HPSEC (B, C).

Fig. 2 Distribution of polar compounds in ROPO/RCO (80:20) after 60 sessions of frying based directly on HPSEC (A) and on combination of solid-phase extraction (SPE) and HPSEC (B, C).

Fig. 3 Tocopherol chromatogram of the ROPO pure before frying obtained by GC-FID. 


\section{Figure 1}

$\mathbf{A}$
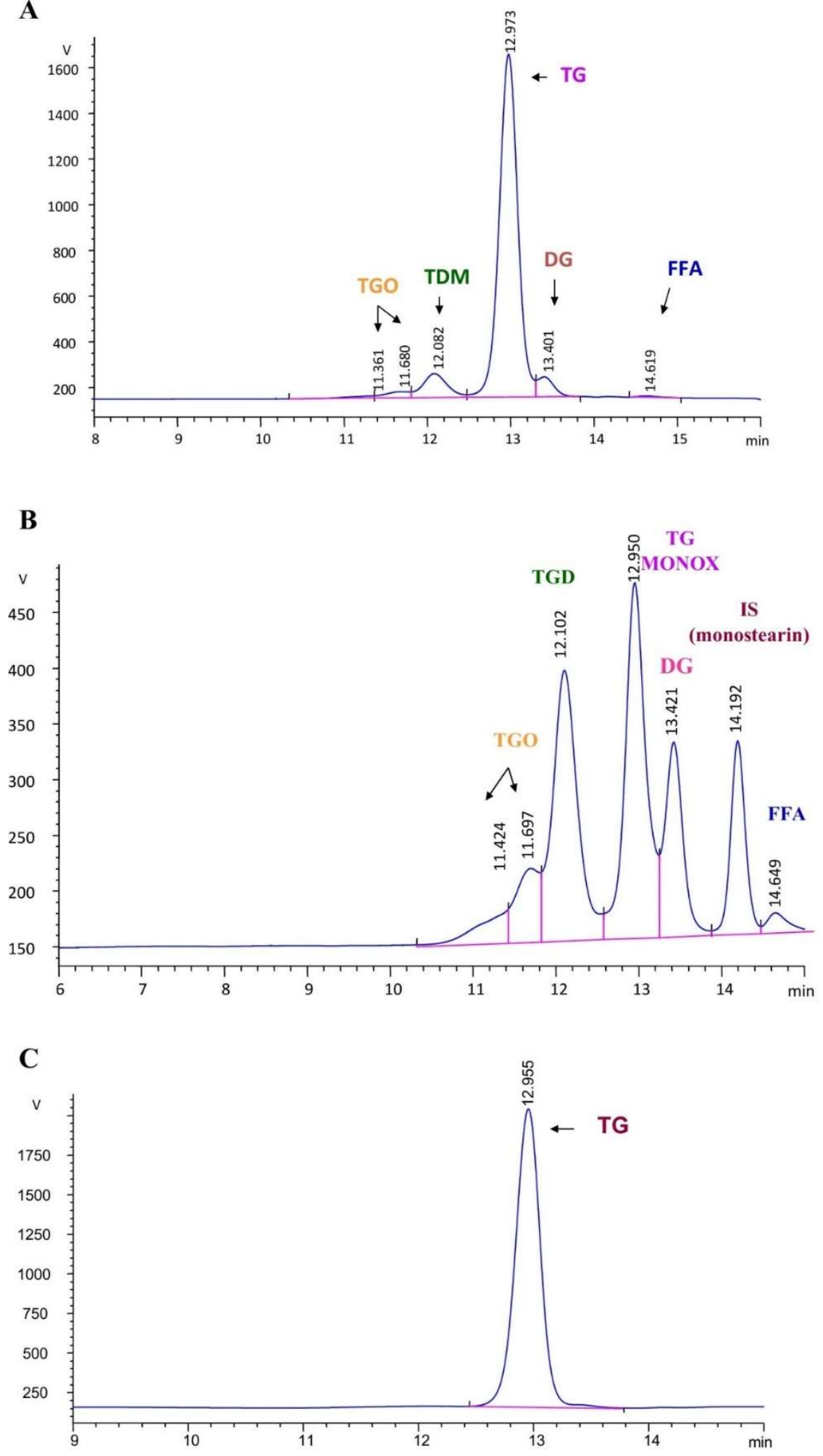

Eur Food Res Technol 245, 967-976 (2019). https://doi.org/10.1007/s00217-018-3202-5 
Figure 2
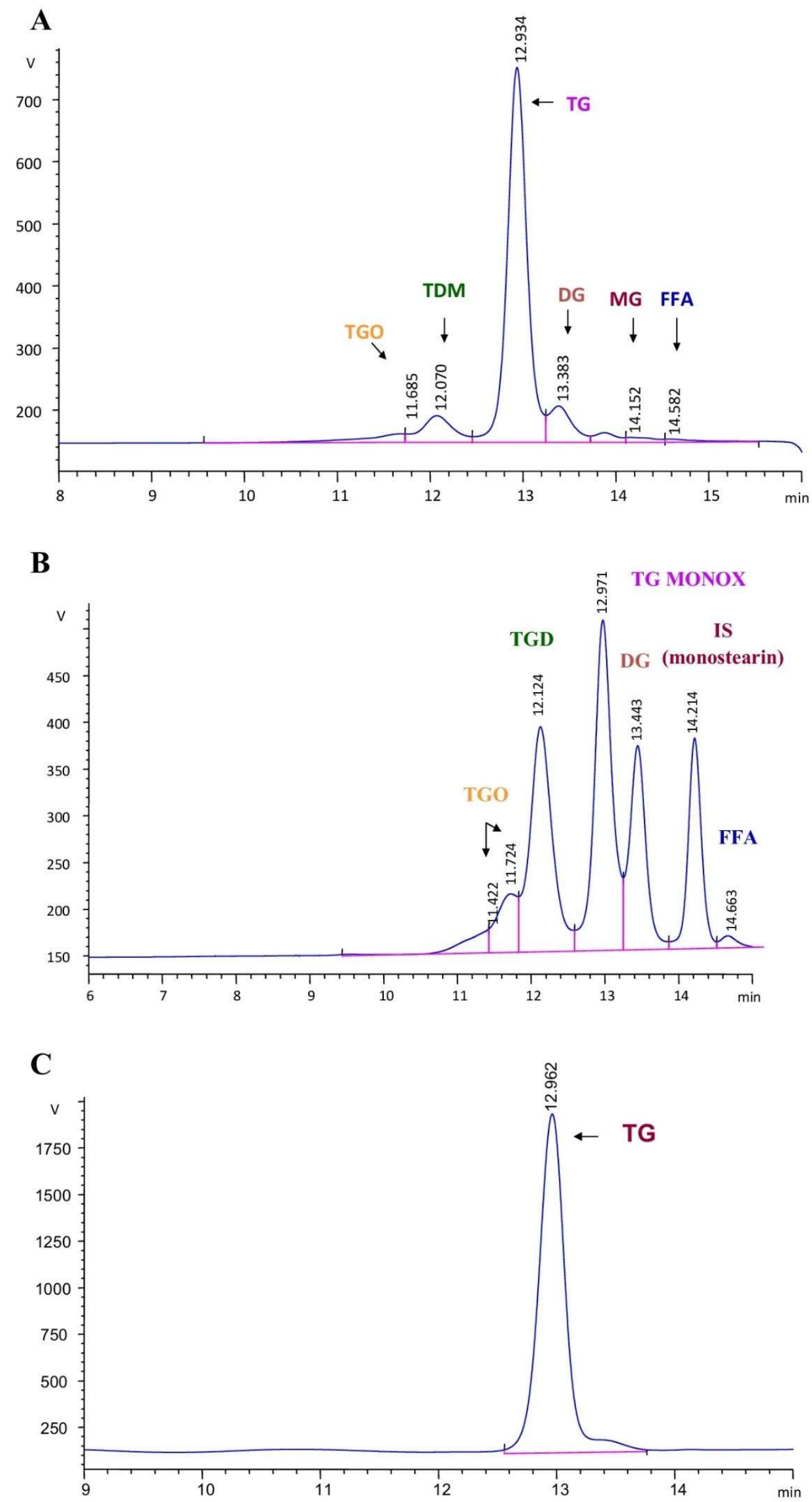

Eur Food Res Technol 245, 967-976 (2019). https://doi.org/10.1007/s00217-018-3202-5 


\section{Figure 3}

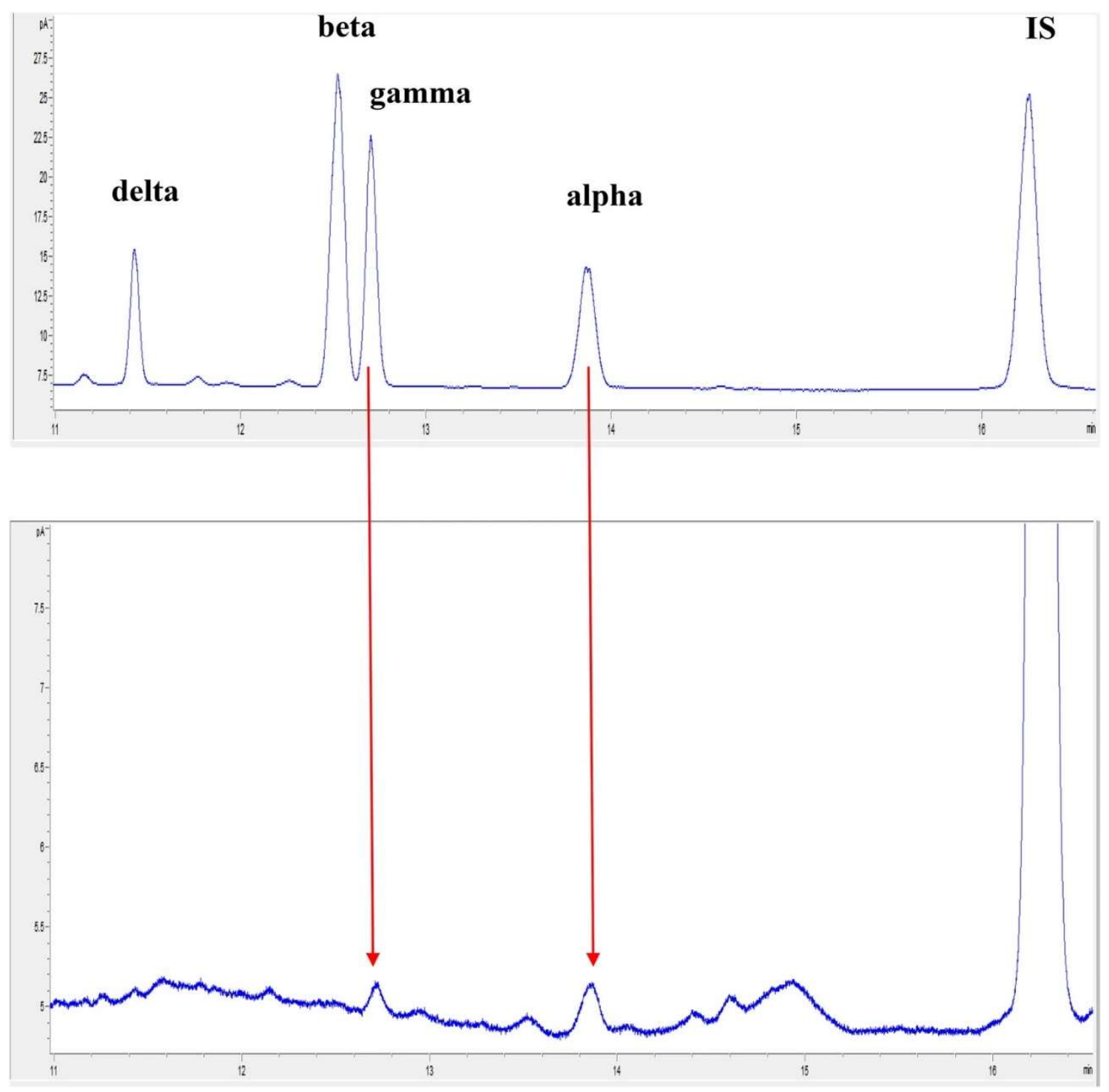

